\title{
Educação Remota em Tempos de Pandemia: Reflexões no Contexto Acadêmico
}

\author{
Remote Education in Times of Pandemic: reflections in the academic \\ context
}

Valdes Roberto Bollela1 (D), Igor Studart Medeiros² (D), Simone Telles ${ }^{3}$ (D)

\begin{abstract}
The Covid-19 pandemic resulted in the suspension of face-to-face educational activities at all levels, from basic to higher education. Following the recommendations of the Ministry of Health and the São Paulo State Department of Health and without being able to maintain the usual teaching activities, students, teachers, and specialists had the opportunity to experience a new educational reality that had the technology and distance learning strategies as the main options available for maintaining the teaching and learning process throughout 2020. This article shares reflections, strategies and actions focusing on the potentialities of distance learning and remote education (RE). First, we'Il present principles of teaching, learning and student assessment. The discussions did not fall on the use of technology and possible resources that can be used in the classroom, but on the possibilities of transformation of educational programs based on good practices, whether in face-to-face, distance or hybrid education. The article also discusses faculty development and scholarship aspects inside the institutions. Aspects related to technology, infrastructure, human resources, and accessibility to distance education are emphasized. We also approach Virtual Learning Environments (VLE) as places that allow the provision of content and learning management; student assessment; review of institutional policy and rules of distance education as mechanisms that can ensure the pedagogical and curricular organization of the courses offered.
\end{abstract}

Keywords: Higher, education; Remote, education; Education, Distance; Covid-19

\section{RESUMO}

A pandemia da Covid-19 e as ações para seu enfrentamento resultaram, entre outras coisas, na suspensão das aulas presenciais nas instituições de ensino e em todos os níveis, da educação básica ao ensino superior. Seguindo as recomendações do Ministério da Saúde e da Secretaria Estadual da Saúde e sem poder manter as atividades de ensino presenciais, estudantes, professores e especialistas da área tiveram a oportunidade de vivenciar uma nova realidade educacional que teve a tecnologia e recursos de educação a distância (EaD) como as principais ferramentas disponíveis para manutenção do processo de ensino e aprendizagem ao longo do ano de 2020 . Este artigo tem como objetivo compartilhar reflexões sobre ações e estratégias utilizadas no ensino superior, focando as potencialidades EaD e da educação remota em sua modalidade emergencial (ERE). A partir do impacto resultante da pandemia, apresentaremos neste artigo alguns princípios e aspectos relevantes e necessários aos processos de ensino, aprendizagem e avaliação de estudantes. As discussões não recaíram sobre o uso da tecnologia e possíveis recur-

\footnotetext{
1. Universidade de São Paulo, Faculdade de Medicina de Ribeirão Preto (FMRP-USP), SP, Brasil

2. Universidade de São Paulo, Faculdade de Odontologia (FO-USP), SP, Brasil

3. Universidade Virtual do Estado de São Paulo (UNIVESP), SP, Brasil
} 
sos que podem ser utilizados em sala de aula, mas sim sobre as possibilidades de transformação dos programas educacionais com base nos princípios que regem as boas práticas, seja no ensino presencial, a distância ou híbrido. Destacam-se, neste artigo, discussões a respeito dos princípios básicos na organização da atividade educacional; desenvolvimento docente e mérito acadêmico a partir de treinamento docente para o uso de novas estratégias e tecnologias de ensino e avaliação; infraestrutura, recursos humanos e acessibilidade para EaD ressaltando a importância da disponibilização de recursos e infraestrutura básica de TI para apoiar atividades mediadas por tecnologia. Também abordamos os Ambientes Virtuais de Aprendizagem (AVA) como espaços que permitem oferecimento de conteúdo e gestão da aprendizagem; avaliação dos estudantes; revisão da política institucional, normas e regras da EaD enquanto mecanismos que podem zelar pela organização pedagógica e curricular dos cursos ofertados.

Palavras-chave: Ensino superior, Educação remota, Educação a distância, Covid-19

\section{MENSAGENS PRINCIPAIS:}

- A pandemia da Covid-19 tem muitos significados, e vários não muito bons. No entanto é inegável que representa uma oportunidade única para rever $\mathrm{e}$ melhorar as práticas educacionais no ensino superior;

- Valorizar o trabalho docente, no ensino de graduação e pós-graduação, é a forma de manter a criatividade e vitalidade destes profissionais, além do interesse pela busca da excelência nesta área estruturante de toda universidade;

- Desenvolvimento docente para o ensino é uma das formas de valorizar o papel docente na universidade;

- Atenção ás normas e regulamentações é fundamental para dar segurança à revisão de processos educacionais.

\section{CONTEXTO:}

No final de 2019, tivemos as primeiras notícias de uma nova doença viral e do aumento do número de casos na China. A Covid-19 é uma doença respiratória de alta transmissibilidade, causada por um novo coronavírus, que viria a ser denominado, alguns meses depois, SARS-CoV-2, e que mudou radicalmente a vida das pessoas em todo o planeta ${ }^{1}$. Em 26 de fevereiro, foi confirmado o primeiro caso da doença no Brasil, na cidade de São Paulo e a partir de março, segundo recomendações do Ministério da Saúde e da Secretaria de Estado da Saúde, as instituições de ensino superior (IES) de todo o Brasil decidiram pela suspensão das aulas presenciais.

O caráter emergencial da medida amplificou enormemente os desafios, pois a educação mediada por tecnologia não era usada em larga escala na maioria das IES e, da noite para o dia, teve de ser implementada. A pandemia teve e ainda tem causado inúmeros reflexos na forma das pessoas se relacionarem, trabalharem, aprenderem e se divertirem. No campo da educação, os reflexos atingiram todos os níveis, com impactos significativos na educação fundamental e ensino médio, especialmente entre as populações mais vulneráveis e sem acesso a recursos de tecnologia da informação e comunicação (TIC) e conectividade. No entanto, o foco deste artigo está direcionado ao impacto da pandemia, as reflexões e ações que tivemos de tomar no contexto do ensino superior.

A situação de enfrentamento da Covid-19, após a suspensão das aulas, nos permitiu identificar fragilidades e potencialidades para a educação a distância (EaD) na medida em que fazíamos educação remota emergencial (ERE) durante praticamente todo o ano de 2020. Esta jornada foi e continua sendo uma oportunidade para rever processos e de aprendizagem para o ensino superior, não só em termos de uso das TIC, mas também em metodologias que possam abarcar a nova realidade educacional que estamos vivenciando.

A maior parte destas reflexões foram feitas a partir de conversas entre os autores e um grupo de professores e especialistas da área, aos quais registramos nosso agradecimento. Optamos por utilizar o termo "educação" e não "ensino", pois expressa melhor os processos multidirecionais presentes na interação que resulta na aprendizagem das pessoas (estudantes, professores, outros atores), além do fato de que os decretos do Conselho Estadual de Educação (CEE) e do Ministério da Educação e Cultura (MEC) que esta- 
belecem diretrizes e bases para esta modalidade utilizam o termo "educação".

O termo EaD é usado para designar experiências educacionais que foram planejadas para execução na modalidade não presencial, usando recursos tecnológicos, com atividades executadas de forma síncrona e/ou assíncrona, incluindo aulas gravadas. A educação remota tem sido usada para indicar a mudança temporária do ensino presencial ou híbrido, para uma modalidade totalmente não presencial e mediada por algum tipo de TIC.

Mesmo com todas as dificuldades e sofrimento impostos pela necessidade de adaptação à ERE, temos vivido e ouvido inúmeros relatos de professores e estudantes que descobriram novas possibilidades e potencialidades para alcançar objetivos de aprendizagem propostos nas disciplinas. Muito antes da pandemia, já sabíamos que o processo educacional deve estar centrado naquele que aprende e não naquele que ensina ou nas tecnologias/estratégias de ensino, sejam elas ofertadas de maneira presencial ou a distância.

Percebemos também que, apesar de focarmos no ensino de graduação e nos cursos de extensão, a ERE e a EaD permitem também viabilizar atividades da pós-graduação, de pesquisa, extensão e da gestão. Temos aprendido isso no dia a dia, na medida em que transformamos processos antes exclusivamente presenciais em atividades síncronas mediadas por alguma TIC. Ao sermos obrigados a adotar estes recursos de forma intensiva, percebemos seu potencial de ampliação de acessibilidade e sua viabilidade. Como mencionado anteriormente, este artigo não busca contrapor educação presencial com ERE ou EaD, mas é um exercício que busca avançar as fronteiras do conhecimento e das possibilidades de uso integrado e articulado destes recursos criando oportunidades reais de crescimento pessoal e profissional para todos os envolvidos.

Neste sentido, apresentaremos alguns aspectos relevantes que são essenciais para o planejamento e aprimoramento dos processos de ensino, aprendizagem e avaliação de estudantes e programas educacionais. Eles foram organizados para facilitar a compreensão do que é real- mente importante para alcançarmos resultados (desfechos) satisfatórios em termos da formação profissional, de qualidade, no ensino superior. Optamos por dar ênfase, inicialmente, ao que entendemos ser estruturante para uma experiência educacional de alto nível e que cumpra com os objetivos e propósitos previamente estabelecidos. Reconhecendo a importância de todos os elementos e aspectos que serão tratados, começamos pelos princípios educacionais universais que regem as boas práticas. É o "core", ou cerne comum, que precisa ser reconhecido e praticado pelos professores em favor do processo de ensino e aprendizagem.

\section{COMPONENTE EDUCACIONAL: PRINCÍPIOS}

Não importa se falamos de educação presencial, a distância ou híbrida, sempre será necessário que o professor siga princípios básicos na organização de sua atividade educacional, a começar pela elaboração e divulgação do plano de ensino e aprendizagem (PEA) que deve conter informações claras e precisas sobre os resultados esperados em termos de aprendizagem e os meios que serão utilizados para ajudar os estudantes a alcançá-los. Normalmente estes resultados são descritos na forma de objetivos de aprendizagem para os componentes cognitivo, psicomotor e atitudinal a serem demonstrados pelos estudantes. Alguns currículos definem adicionalmente as competências para a prática profissional.

Atividades como aulas teóricas, seminários, vídeo aulas, estudo dirigido, discussão em grupo e práticas profissionalizantes são apenas meios, utilizados pelo professor, para auxiliar os estudantes a alcançarem os resultados previamente estabelecidos. Ao listar e priorizar objetivos de aprendizagem, o professor tem condições de definir quais são as melhores estratégias, dentre as disponíveis para serem utilizadas. Este mesmo raciocínio deve ser utilizado para a avaliação do estudante, sempre guiado pelos objetivos de aprendizagem (Figura 1) 


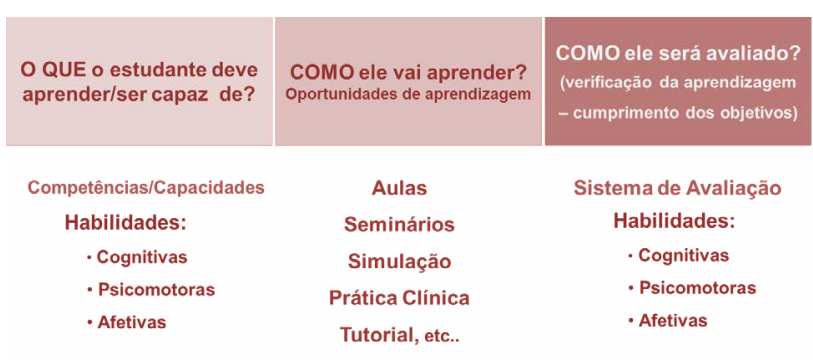

Fonte: Autores.

FIGURA 1: Alinhamento entre objetivos de aprendizagem, estratégias de ensino e avaliação do estudante presentes no plano de ensino e aprendizagem.

Um fato interessante que veio à tona logo nas primeiras semanas da pandemia, quando parte dos professores migravam para a ERE foi a avalanche de atividades e avaliações que foram propostas aos estudantes, sem considerar o momento inusitado que também estava sendo vivido por eles. Os estudantes logo reclamaram, solicitando aos docentes a revisão destas práticas pois criavam uma sobrecarga de trabalho nem sempre necessária ou compatível com o tempo disponível, além de gerar estresse emocional e físico ${ }^{2}$. Esta situação apenas chamou a atenção para algo que já acontecia em boa parte dos cursos superiores no Brasil, a sobrecarga de conteúdo teórico, muitas vezes descontextualizada e não priorizada.

Professores, de um modo geral, valorizam muito o que ensinam e tendem a superdimensionar os objetivos de aprendizagem das suas disciplinas, mesmo que eles são estejam explícitos no plano de ensino das mesmas. De tudo o que sabemos sobre cada tópico que ensinamos, existe muito conteúdo interessante e importante, mas dependendo de cada grupo de alunos (iniciantes ou mais avançados), existirá um conteúdo que é essencial para aquele público, no contexto de cada curso. Definir objetivos de aprendizagem que contenham apenas o essencial é uma das nobres tarefas do professor e permite liberar tempo e espaço para atividades que não envolvam apenas aulas teóricas e memorização (aprender por aprender). Espera-se que as atividades educacionais busquem dar o sentido ao que está sendo aprendido (aprender para compreender e ser capaz de aplicar) utilizando estratégias que dão maior protagonismo ao estudante tais como de- bates, diálogos, discussão de problemas e temas ligados à prática profissional. Neste cenário, vale a máxima de que "MENOS É MAIS".

Em resumo, o professor deve elaborar o um plano de ensino com a definição clara do conteúdo essencial, na forma de objetivos de aprendizagem, e deve sempre comunicá-lo claramente aos estudantes. Esta tarefa é bastante facilitada quando utilizamos ambientes virtuais de aprendizagem (AVA) que podem facilitar o acesso e a recuperação destes materiais para sua atualização, facilitando o trabalho dos professores. A partir dos objetivos de aprendizagem definidos, seguese a escolha das estratégias de ensino e aprendizagem mais adequadas aos propósitos definidos e alinhadas à avaliação do estudante que deve conter um componente formativo (avaliação que induz e promove aprendizagem) e um componente somativo que será elemento importante na tomada de decisão sobre a progressão do estudante.

\section{DESENVOLVIMENTO DOCENTE PARA EDUCAÇÃO E MÉRITO ACADÊMICO}

Assim como na educação presencial, na EaD existem inúmeras estratégias de ensino e de avaliação disponíveis. Um aspecto decisivo no sucesso da estratégia está pautado no domínio e familiaridade do professor com esses diferentes recursos de ensino. Neste sentido, as instituições precisam criar oportunidades de treinamento para os professores interessados em utilizar estas novas estratégias de ensino e avaliação. A criação de instâncias formais de apoio ao docente e à docência, através de unidades ou centros de desenvolvimento docente (do inglês Faculty Development Units) ou Centros de Apoio ao Ensino e Aprendizagem (Centers for Teaching and Learning) são opções interessantes e viáveis ${ }^{3,4}$. Na Universidade de São Paulo, algumas unidades já contam com centros de desenvolvimento docente para o ensino, tais como a Faculdade de Medicina de Ribeirão Preto (FMRP) (http://cdde.fmrp.usp.br/) e a Faculdade de Medicina (https://www.fm.usp.br/cedem/portal/). A Universidade conta também com grupos de apoio pedagógico (GAP) e mais recentemente, uma Comissão de Formação Didático Pedagógica na Pró Reitoria de Pós-Graduação. 
Partindo do pressuposto de que nenhum professor recém contratado pode ser considerado completamente preparado para a docência ou para a pesquisa, é fundamental que exista estímulo, ao longo da vida profissional, para o desenvolvimento destas competências requeridas para exercer a função docente no mais alto nível. Todavia, a valorização existente nas universidades públicas brasileiras para progressão na carreira tende a estimular investimento na pesquisa, em detrimento a atuação na docência. Se houver um desequilíbrio muito grande entre o valor atribuído à pesquisa quando comparada ao valor atribuído ao ensino (graduação e pós-graduação), isto terá consequências indesejadas e impactará na disponibilidade e interesse dos docentes para aquisição de das habilidades voltadas à docência.

\section{INFRAESTRUTURA, RECURSOS HU- MANOS E ACESSIBILIDADE PARA EAD}

Durante as primeiras semanas da pandemia, ficou evidente a importância de garantir infraestrutura básica de TIC, que inclui hardwares e softwares em quantidade e qualidade suficientes para apoiar este tipo de atividade. Todas as instituições de ensino brasileiras foram desafiadas pela pandemia do novo coronavírus a testarem sua capacidade operacional. Lembrando que estes recursos precisam estar disponíveis para o ensino nas modalidades síncrona e assíncrona, 24 horas por dia, todos os dias da semana.

$A$ acessibilidade dos estudantes à internet de banda larga e equipamentos minimamente adequados para o desenvolvimento das atividades remotas foi outro grande desafio. Dentre as medidas que poderiam ser implementadas neste campo, a partir da situação vivida durante a primeira onda da pandemia da Covid-19, destacamos: identificação sistemática e regular das necessidades específicas dos estudantes por meio de ações articuladas com o Serviço Social; o planejamento financeiro e logístico para o fornecimento deste acesso de acordo o grau de dificuldade de acesso aos equipamentos ou serviços; a definição do setor responsável pela manutenção e gestão dos equipamentos e kits de acesso. Acreditamos que o investimento na universalização do acesso a EaD tem o potencial de modificar, no futuro, as disciplinas estritamente presenciais permitindo novas abordagens pedagógicas, incluindo realização de avaliações presenciais usando os computadores disponibilizados ou dos próprios estudantes.

\section{AMBIENTES VIRTUAIS DE APRENDI- ZAGEM}

A pandemia tem sido um catalisador para que professores conheçam e se apropriem dos recursos disponíveis para ER e EaD em suas instituições. Imediatamente após a suspensão das aulas presenciais, foram deflagradas algumas orientações genéricas para que cada professor encontrasse os meios para manter as atividades e literalmente "sobreviver" ao mesmo tempo que cuidava de inúmeras outras demandas, de ordem pessoal e profissional.

Dentre os diversos AVA disponíveis no mercado destacamos alguns que têm uso disseminado tanto em nível nacional quanto internacionalmente: Moodle, Blackboard, Canvas, Google classroom, Microsoft teams. Os três primeiros são plataformas voltadas à educação e os outros são ambientes corporativos que foram rapidamente adaptados para educação remota na pandemia. O que difere algumas destas soluções geralmente é o custo, a possibilidade de customização, sistema de gestão da aprendizagem (pacote fechado ou recurso aberto).

Os métodos e estratégias de ensino e aprendizagem disponíveis podem ser agrupados de acordo com suas características para compreendermos seus recursos e principalmente as suas indicações e potencialidades para auxiliam os estudantes a alcançarem os objetivos propostos e resultados esperados. A seguir, listamos algumas das estratégias de ensino de acordo com a atividade que faremos no AVA:

Oferecimento de conteúdo (aulas, textos, vídeos demonstrativos): Aulas: podem ser síncronas (em tempo real) e assíncronas (gravadas e disponibilizadas por longo período). Para ministrar as aulas em tempo real ou gravá-las, vários são os programas disponíveis. Alguns dos recursos mais utilizados na pandemia são o Zoom $^{\circledR}$, GotoMeeting ${ }^{\circledR}$ e o Google Meet $^{\circledR}$. Estas ferramentas permitem a realização de reuniões com estudantes (tira-dúvi- 
das e debates), aulas síncronas, assim como a gravação dos momentos presenciais para disponibilização posterior. $O$ recurso conferência Web, também conhecido como Mconf, é uma ferramenta semeIhante e pode ser acessada utilizando a Comunidade Acadêmica Federada (CAFe) e no Brasil ela está disponível no pacote do Moodle. A interação pode ser planejada no próprio software (MConf, Zoom, etc...) ou através de aplicativos específicos como o Edpuzzle ${ }^{\circledR}$, Vizia $^{\circledR}$ e outros. Se o docente preferir fazer uma aula mais interativa ele pode intercalar atividades durante a aula usando outras ferramentas como o questionário interativo do Moodle, formulário do Google, Kahoot ${ }^{\circledR}$ ou Mentimeter ${ }^{\circledR}$.

Vídeos e textos: respeitados os direitos autorais, vídeo aulas, textos jornalísticos e científicos e vídeos de plataformas externas (e.g. Youtube) podem ser disponibilizados no AVA da instituição na forma de uma tarefa, seguida de instruções para os estudantes. Aqui seria o local onde o professor pode anexar também a sua própria aula gravada.

A comunicação regular com os estudantes é outro elemento chave para o sucesso das atividades desenhadas para o ambiente virtual. 0 aprendizado ativo pressupõe também a realização de atividades pelos estudantes e a comunicação com o professor, criando uma comunidade virtual de aprendizagem. Os recursos listados a seguir estão todos disponíveis no Moodle (e em outras plataformas) e podem ser utilizados pelos professores para potencializar as oportunidades de aprendizagem: enquete (opinar e dar retorno às propostas educativas); questionário (elaborar questões e prover feedback); tarefas (realizar um "trabalho" no AVA e fora dele).

Algumas ferramentas disponíveis no Moodle são facilitadoras da comunicação entre estudantes e destes com o professor, como o fórum para comunicação por texto, voz e vídeo de maneira assíncrona entre os participantes da disciplina ou por grupos previamente divididos pelo professor.

\section{AVALIAÇÃO DO ESTUDANTE:}

Considerando os diferentes propósitos da avaliação do estudante os AVA são muito úteis para a avaliações formativas, ou melhor dizendo aquelas que PROMOVEM a aprendizagem. A proposta de atividades a serem desenvolvidas pelos estudantes pode ser estimulada e acompanhada no AVA facilmente, e com a enorme vantagem de ficarem registradas com a possibilidade de se oferecer devolutiva que ficam todas registradas e acessíveis. Os AVA permitem a criação de bancos de questões e a geração de questionários. Isso facilita muito a avaliação somativa e formativa no âmbito da disciplina.

É muito importante planejar a disciplina, já prevendo as atividades que serão utilizadas como indicadoras da participação do estudante, e consequentemente servirão como oportunidade para prover devolutivas e checagem da aprendizagem. Ao adotar esta conduta, o docente cria alternativas viáveis de avaliação que tem como principal finalidade promover a aprendizagem e não apenas decidir sobre quem será aprovado ou reprovado (avaliações somativas). Durante a pandemia, muitos professores tentaram realizar avaliações nos moldes que faziam no presencial. Via de regra, essas tentativas fracassaram. A avaliação no contexto da ER e da EaD deve ser revista e reprogramada, fortalecendo aspectos formativos, com maior frequência, menor quantidade de conteúdo e a realização de devolutivas regulares ${ }^{5,6}$.

Outro aspecto que difere atividades em EaD daquelas realizadas em disciplinas presenciais é a forma de computar e valorizar a presença dos estudantes. Recomenda-se que, no lugar da presença utilizada em aulas presenciais, o professor utilize a participação do estudante nas atividades, fóruns, questionários, como forma de evidenciar sua participação.

Alguns recursos disponíveis no Moodle podem auxiliar na percepção do avanço do estudante no AVA, como a utilização da barra de progresso das atividades, assim como a identificação do tempo de permanência do estudante no AVA.

Resumos das estratégias que favorecem a ERE e a EaD efetiva:

1. Estabelecimento de uma rotina de horário para oferta de atividades síncronas;

2. Comunicação frequente do docente com os estudantes nos fóruns e através de mensagens. Isto permite que os estudantes se sintam "acolhidos" e afasta a percepção que estão "abandonados"; 
3. Utilização de enquetes para "sentir" a percepção dos estudantes sobre o curso (avaliação da disciplina);

4. Produção de vídeos mais curtos que aqueles que costumamos fazer presencialmente;

5. Intercalar as aulas com vídeos demonstrativos, textos, e afazeres para os estudantes, como: pesquisas, questionários ou outras produções;

6. Estimular a participação dos estudantes nos fóruns. Os estudantes podem aprender uns com os outros e tirar dúvidas com a criação de uma verdadeira comunidade virtual de aprendizagem;

7. Dar o "feedback" sobre atividades e respostas às perguntas dos estudantes nos fóruns e tira-dúvidas. Isso indica o comprometimento do docente com o aprendizado do estudante.

\section{REVISÃO DA POLÍTICA INSTITUCIO- NAL, NORMAS E REGRAMENTO PARA EAD}

Para criar e ampliar estratégias de EaD na graduação e pós-graduação em uma instituição de ensino superior, o estabelecimento de uma política educacional é essencial para organização pedagógica e curricular dos cursos a serem ofertados, fazendo-se necessária a atualização ou a criação de portarias e normas que regulamentam as atividades propostas, sempre em consonância com as regulamentações do Conselho Estadual de Educação do Estado de São Paulo (CEE-SP) e Ministério da Educação (MEC). Há de se considerar duas vertentes quando o assunto é EaD: a oferta de cursos totalmente a distância e a carga horária em EaD nos cursos presenciais (ensino híbrido). No primeiro caso, é necessário o credenciamento institucional nos termos das legislações federais e no segundo devemos atentar de fato à elaboração de políticas instituições, respeitando normas e regras das legislações federais e estaduais.

Neste momento de pandemia, a legislação, a partir da publicação das Portarias MEC no 343/2020, 345/2020, 473/2020, 356/2020, $544 / 2020$ e $1.030 / 2020$ e Deliberações CEE no $177 / 2020$, 181/2020, 185/2020 emergenciais, tem buscado estabelecer medidas educacionais que possam dispor sobre a realização de atividades e procedimentos de avaliação de estudantes com o objetivo de articular o projeto pedagógico institucional ao período de isolamento social devido a pandemia da Covid-19. Muitas instituições iniciaram o ano de 2020 com a publicação de normas para substituição das disciplinas presenciais, em cursos regularmente autorizados, por atividades letivas com a utilização de meios digitais e avaliação de estudantes a distância ${ }^{7-15}$.

Em virtude do cenário criado pela pandemia, incertezas continuam rondando nossa prática pedagógica e medidas emergenciais continuam sendo tomadas, tais como a publicação, em dezembro de 2020, de normas que dispõem sobre retorno às aulas presenciais e sobre caráter excepcional de utilização de recursos educacionais digitais, observando regras locais de distanciamento social e o Protocolo de Biossegurança do MEC instituído em julho de 2020. Desde o início da pandemia temos vivenciado mudanças, as vezes pouco coerentes e até contraditórias, o que tem pressionado muito as instituições e gestores que nem sempre tem clareza do que fazer para atender, ao mesmo tempo, as necessidades educacionais e se adequar às normas.

Em termos de legislação, vivemos um momento que requer uma reavaliação das políticas educacionais institucionais de curto, médio e longo prazos. Em curto prazo, a política institucional segue atrelada aos resultados da pandemia e às normas de isolamento social propostas pelos órgãos públicos federais e estaduais. Em médio e longo prazos a legislação vigente para oferta de cursos presenciais precisam ser revisitadas e caminharmos para o estabelecimento de uma nova política educacional. As instituições de ensino superior do sistema estadual de educação de São Paulo podem ofertar até $20 \%$ da carga horária de seus cursos em EaD, enquanto as federais podem chegar até $40 \%$ de oferta. Neste contexto, as discussões para estabelecimento de bases para criação de uma política institucional precisam versar sobre estratégias didáticas que a instituição pretende adotar, isto é, sobre o tipo de oferta de atividades educacionais que podem se valer de combinações das atividades parcialmente ou totalmente a distância. 
Por exemplo, para viabilizar uma disciplina de recuperação na modalidade $\mathrm{EaD}$ seria preciso que se fizessem ajustes nas normas/regulamentos de graduação permitindo que este tipo atividade fosse possível de ser realizada na instituição e no projeto pedagógico de curso com a formalização do tipo de oferta em termos estratégicos e respectivos percentuais de carga-horária, respeitando às especificidades das Diretrizes Curriculares Nacionais (DCN). O alinhamento entre as ações e o respaldo jurídico e normativo é fundamental e poderá facilitar o trâmite e adesão de professores para este tipo de recursividade nos cursos de graduação e pósgraduação de uma instituição de ensino superior.

Outro aspecto muito importante, que precisa ser pontuado no estabelecimento da política educacional para ampliação de estratégias EaD, é a questão dos direitos autorais, de propriedade intelectual e de segurança dos materiais produzidos. Apesar de ser um tema universal e controverso, ele não foi um impeditivo para que as melhores instituições do mundo criassem e disponibilizassem cursos no formato EaD ou na modalidade de educação híbrida. Muitos destes cursos são oferecidos para professores, pós-graduandos e estudantes de graduação em uma parceria educacional. Portanto, cabe à instituição estabelecer normas e regras para o compartilhamento de conhecimento, considerando que um material didático elaborado por um docente para EaD pode ser disponibilizado dentro e fora da instituição.

A pandemia de Covid-19 trouxe mudanças significativas nas relações sociais por conta do isolamento. A tecnologia entra neste cenário como a grande protagonista, isto é, como um recurso que possibilita a comunicação entre as pessoas seja no âmbito pessoal, profissional ou acadêmico independente da distância geográfica. Há de se ressaltar que a despeito do caráter comunicativo e integrador, o uso emergencial das TIC no momento de isolamento social causou impactos significativos na educação. Por um lado, temos populações mais vulneráveis que não possuem acesso a estes recursos, por outro as instituições de educação básica e ensino superior que tiveram que adaptar estrutura física e estratégias didáticas para dar continuidade ao ano letivo.

Num cenário de muitas incertezas, instituições de ensino tiveram a árdua tarefa de compre- ender as recomendações do Ministério da Saúde e da Secretaria de Estado da Saúde, diretrizes do Conselho Estadual de Educação (CEE) e do MEC para formalizar suas próprias políticas de ensino e aprendizagem de modo ágil. O estabelecimento de tais políticas, de forma geral, oficializou a continuidade dos estudos mediado pela tecnologia, trazendo à tona discussões a respeito das modalidades de ER e EaD 7-15.

As instituições em geral, tiveram um tempo relativamente curto para estabelecer e implantar políticas que pudessem de alguma forma manter em funcionamento atividades essenciais para uma população em confinamento. Os resultados, num primeiro momento, trouxeram dúvidas quanto à sua eficácia, todavia o uso da tecnologia não tem sido considerado um fator prejudicial à experiência educacional e nem tampouco algo que tenha reduzido a qualidade do ensino.

A despeito da grande maioria dos estudantes terem nascido imersos em contextos em que a tecnologia permeia as atividades cotidianas, suas experiências educacionais eram predominantemente presenciais até o início da pandemia.

Independentemente do uso das TICs, o processo de ensino-aprendizagem precisa ser revisitado pelos docentes e o foco precisa recair sobre o planejamento de aulas, sobre o qual os recursos tecnológicos aparecem como ferramentas facilitadoras. Remotamente, a distância ou presencialmente, o equilíbrio entre atividades teóricas e práticas que possam resultar em debates e discussões não pode se restringir ao uso da tecnologia. 0 cerne da questão está em atividades significativas que evitem a sobrecarga de conteúdos demasiadamente teóricos e descontextualizados, e usando a tecnologia a favor do processo de ensino e aprendizagem. È necessário também garantir uma avaliação dos estudantes com forte componente formativo (avaliação que induz e promove aprendizagem) complementando o componente somativo que está mais voltado para a tomada de decisão.

\section{COMENTÁRIOS FINAIS:}

Muitos são os questionamentos sobre como será a educação na pós-pandemia, quais os impactos causados pelo uso da tecnologia no ensino 
e aprendizagem, como se definirão a legislação e as recomendações dos órgãos regulatórios. O cenário ainda não se desenhou por completo em virtude da urgência sanitária e de protocolos que não sejam emergenciais e que possam estabelecer ou não o retorno às atividades presenciais. Neste cenário, entendemos que as instituições de ensino superior precisam estabelecer uma política interna sobre as modalidades de educação que pretendem implantar, seja ERE ou EaD.

O uso institucional da tecnologia precisa ser pautado em estratégias que possam democratizar o ensino, respeitar o uso dos direitos autorais, de propriedade intelectual e de segurança dos materiais produzidos, garantir uma política de educação e de mérito acadêmico com oportunidades de treinamento para os professores, alunos e funcionários interessados em aprender e utilizar novas estratégias e tecnologias de ensino.

Por fim, concluímos que este momento de transição pode ser visto como uma oportunidade que as instituições de ensino possuem para refletir sobre suas políticas internas, prática de ensino e uso de tecnologia como uma recursividade que amplia a disseminação de conhecimento a partir da disponibilização de conteúdo, da interação entre alunos, professores, pesquisadores e comunidade educacional, independente de espaço geográfico e temporalidade. Ações que até o presente momento, foram utilizadas com maior exclusividade nas atividades que fomentam a ampliação e o estreitamento entre pesquisadores do Brasil e do mundo de maneira rápida e com baixíssimo custo.

\section{REFERÊNCIAS}

1. Aquino EML, Silveira IH, Pescarini J, Aquino R, Souza Filho JA. Medidas de distanciamento social no controle da pandemia de COVID-19: potenciais impactos e desafios no Brasil. Ciência e Saúde Coletiva. 2020. 25(SI), 2423-46.

2. Maia BR, Dias PC. Ansiedade, depressão e estresse em estudantes universitários: o impacto da COVID-19. Estudos de Psicologia. 2020. 37, 1-8.

3. Benito A, Green NA, Popely DR, Thai-Garcia PM, Schneiderheinze AT. Developing Faculty to Provide University Students with Improved Learning Experiences. High. Learn. Res. Commun. 2017. 7(2), 1-12.

4. KINZIE J, Landy K, Sorcinelli MD, Hutchings P. Better Together: How Faculty Development and Assessment Can
Join Forces to Improve Student Learning, Change: The Magazine of Higher Learning. 2019. 51(5), 46-54.

5. Dias E, Pinto FCF. Educação e sociedade. Ensaio: Avaliação e Políticas Públicas em Educação. 2019. 27(104), 449-454.

6. Dias E, Pinto FCF. A Educação e a Covid-19. Ensaio: Avaliação e Políticas Públicas em Educação. 2020. 28(108), 545-554.

7. BRASIL. Ministério da Educação. Portaria no 343, de 17 de março de 2020. Dispõe sobre a substituição das aulas presenciais por aulas em meios digitais enquanto durar a situação de pandemia do Novo Coronavírus - COVID-19. Acesso em: $10 \mathrm{fev} .2021$. Disponível em: <https://www. in.gov.br/en/web/dou/-/portaria-n-343-de-17-de-marco-de-2020-248564376>.

8. BRASIL. Ministério da Educação. Portaria no 345, de 19 de março de 2020. Altera a Portaria MEC no 343, de 17 de março de 2020. Acesso em: 10 fev.2021. Disponível em: $<$ https://pesquisa.in.gov.br/imprensa/jsp/visualiza/index.jsp? jornal $=603 \&$ pagina $=1 \&$ data $=19 / 03 / 2020 \&$ totalArquivos $=1>$.

9. BRASIL. Ministério da Educação. Portaria no 456, de 20 de março de 2020. Dispõe sobre a atuação dos alunos dos cursos da área de saúde no combate à pandemia do COVID-19 (coronavírus). Acesso em: 10 fev.2021. Disponível em: <http://www.planalto.gov.br/CCIVIL_03/Portaria/PRT/Portaria\%20n\%C2\%BA\%20356-20-mec.htm>.

10. BRASIL. Ministério da Educação. Portaria no 473, de 12 de maio de 2020. Prorroga o prazo previsto no $\S 10$ do art. $1^{\circ}$ da Portaria no 343, de 17 de março de 2020. Acesso em: 10 fev.2021. Disponível em: <https://www. in.gov.br/en/web/dou/-/portaria-n-473-de-12-de-maiode-2020-256531507? inheritRedirect $=$ true \&redirect $=\%$ 2Fweb $\% 2$ Fguest $\% 2$ Fsearch $\% 3$ FqSearch \% 3DPortaria $\% 2520473 \% 252 \mathrm{C} \% 252012 \% 2520 \mathrm{de} \% 2520$ maio $\%$ 2520de\%25202020>.

11. BRASIL. Ministério da Educação. Portaria no 1030, de 01 de dezembro de 2020. Dispõe sobre o retorno às aulas presenciais e sobre caráter excepcional de utilização de recursos educacionais digitais para integralização da carga horária das atividades pedagógicas enquanto durar a situação de pandemia do novo coronavírus - Covid-19. Acesso em: 10 fev.2021. Disponível em: <https://www.in.gov.br/en/web/dou/-/portaria-n-1.030de-1-de-dezembro-de-2020-291532789>.

12. CONSELHO ESTADUAL DE EDUCAÇÃO (São Paulo). Deliberação no 177, de 19 de março de 2020. Fixa normas quanto à reorganização dos calendários escolares, devido ao surto global do Coronavírus, para o Sistema de Ensino do Estado de São Paulo, e dá outras providências. Acesso em: 10 fev.2021. Disponível em: <http:// cegep.com.br/wp-content/uploads/2020/03/Deliberacao-177-2020-CEE-SP.pdf>.

13. CONSELHO ESTADUAL DE EDUCAÇÃO (São Paulo). Deliberação no 181 , de 15 de junho de 2020. Dispõe sobre a avaliação de estudantes em cursos devidamente autorizados na modalidade EaD. Acesso em: 10 fev.2021. Disponível em: <https://deguaratingueta.educacao.sp.gov. br/resolucao-de-15-6-2020-deliberacao-cee-181-2020- 
dispoe-sobre-a-avaliacao-de-estudantes-em-cursos-devidamente-autorizados-na-modalidade-ead/>.

14. CONSELHO ESTADUAL DE EDUCAÇÃO (São Paulo). Deliberação no 185, de 17 de julho de 2020. Fixa procedimentos para o cumprimento da carga horária de estágio, atividades práticas, atividades laboratoriais e de internato visando a continuidade e conclusão dos cursos da área da saúde nas Instituições de Ensino Superior, vinculadas ao Sistema de Ensino do Estado de São Paulo, tendo em vista o surto global da Covid-19, e dá ou- tras providências. Acesso em: 10 fev.2021. Disponível em: <https://www.fmb.unesp.br/Home/orientacoesparaoretorno/deliberacao-cee-185.2020.pdf>.

15. MINISTÉRIO DA SAÚDE. Secretaria de Atenção Especializada à Saúde Departamento de Atenção Hospitalar, Domiciliar e de Urgência. Protocolo de Tratamento do Novo Coronavírus (2019-nCoV). Brasília DF, 2020. Acesso em: 10 fev.2021. Disponível em: <https://portalarquivos2.saude. gov.br/images/pdf/2020/fevereiro/05/Protocolo-de-manejo-clinico-para-o-novo-coronavirus-2019-ncov.pdf>.

\section{Contribuições dos autores:}

Todos os autores contribuíram igualmente com as ideias e argumentos usados na elaboração deste texto.

\section{AGRADECIMENTOS:}

Danielle Benvenutto; Ewout ter Haar; Fátima de Lourdes dos Santos Nunes Marques e Luis Gustavo Marcassa pelas contribuições discussão dos argumentos e pontos de vista trazidos neste texto.

Nenhuma fonte de financiamento

Corresponding Author:

Igor Studart Medeiros

igorsm@usp.br

Editor:

Prof. Dr. Marcelo Riberto

Recebido: $30 / 04 / 2021$

Aprovado: $13 / 07 / 2021$

cc) (i) Este é um artigo publicado em acesso aberto (Open Access) sob a licença Creative 